\title{
INTERPOLATION SPACES BETWEEN THE LIPSCHITZ CLASS AND THE SPACE OF CONTINUOUS FUNCTIONS
}

\author{
MICHAEL CWIKEL AND MIECZYSEAW MASTYŁO
}

(Communicated by Dale Alspach)

\begin{abstract}
It is shown that the complex interpolation spaces $[C([0,1])$, $\left.\operatorname{Lip}_{1}([0,1])\right]_{\theta}$ and $\left[C([0,1]), \operatorname{Lip}_{1}([0,1])\right]^{\theta}$ do not coincide with $\operatorname{Lip}_{\theta}([0,1])$ or $\operatorname{lip}_{\theta}([0,1])$ and also that the couple $\left(C, L i p_{1}\right)$ is not a Calderón couple. Similar results are also obtained for the couples $\left(C, \operatorname{Lip}_{\alpha}\right)$ and $\left(\operatorname{Lip}_{\alpha}, \operatorname{Lip}_{1}\right)$ when $\alpha \in(0,1)$.
\end{abstract}

\section{INTRODUCTION}

As usual we let $C=C[0,1]$ denote the space of continous functions $f:[0,1] \rightarrow \mathbb{C}$ with $\|f\|_{C}=\sup _{t \in[0,1]}|f(t)|$. We let $\omega(\delta, f)$ denote the modulus of continuity of the function $f:[0,1] \rightarrow \mathbb{C}$, i.e.

$$
\omega(\delta, f)=\sup _{s, t \in[0,1], 0<|s-t|<\delta}|f(s)-f(t)| \text { for each } \delta>0 .
$$

For each $\theta \in[0,1]$, let $\Lambda_{\theta}=\Lambda_{\theta}[0,1]$ (or $\operatorname{Lip}_{\theta}$ ) denote the space of functions $f \in C$ for which the (Lipschitz) norm

$$
\|f\|_{\Lambda_{\theta}}=\max \left\{\|f\|_{C}, \sup _{\delta>0} \frac{\omega(\delta, f)}{\delta^{\theta}}\right\}
$$

is finite. In particular we let $\Lambda=\Lambda_{1}$. Observe also that $\Lambda_{0}=C$ to within equivalence of norms.

The "little" Lipschitz space $\lambda_{\theta}$ (or $\operatorname{lip}_{\theta}$ ) is defined to be the subspace of $\Lambda_{\theta}$ consisting of those functions $f$ which satisfy the condition $\lim _{\delta \rightarrow 0} \frac{\omega(\delta, f)}{\delta^{\theta}}=0$.

Let $S$ be the usual "unit strip" $S=\{z \in \mathbb{C} \mid 0 \leq \operatorname{Re} z \leq 1\}$.

In this note we consider the complex interpolation spaces for the couple $(C, \Lambda)$, i.e. the spaces $[C, \Lambda]_{\theta}$ and $[C, \Lambda]^{\theta}$ defined as in [7]. The $K$-functional for this couple was calculated exactly many years ago by Peetre (see [14], cf. also [3] Proposition 3.1.9, p. 301) and thus the real interpolation spaces for this couple are well known. However the problem of determining the complex interpolation spaces

Received by the editors September 2, 1994.

1991 Mathematics Subject Classification. Primary 46M35, 46E15, 46E35.

Key words and phrases. Lipschitz class, complex interpolation space, Calderón couple.

The research of the first author was supported by the Fund for Promotion of Research at the Technion. The research of the second author was supported in part by a Lady Davis Fellowship at the Technion. 
has remained open. We show that they are not Lipschitz spaces nor little Lipschitz spaces, and also that the couple $(C, \Lambda)$ is not a Calderón couple. This latter result has also been proved by Bychkova [6] using a different argument based on a result announced by Ovchinnikov [13]. We also obtain analgous results for the couples $\left(C, \Lambda_{\alpha}\right)$ and $\left(\Lambda_{\alpha}, \Lambda\right)$ for $0<\alpha<1$.

We refer to [7] and [3] respectively for further general information about complex interpolation spaces and Calderón couples.

We thank Yuri Brudnyi, Leonid Hanin and Alexander Shteinberg for some helpful discussions. Some forthcoming papers by Brudnyi and Shteinberg [4], [5] extend our results here. In [4] the authors consider couples of $\left(\Lambda\left(\omega_{0}\right), \Lambda\left(\omega_{1}\right)\right)$ of Lipschitz spaces where the powers $\delta^{\theta}$ are replaced by more general functions $\omega_{j}$ and give necessary and sufficient conditions for such couples to be Calderón. From the results of [5] it follows that the couple $(C, \Lambda)$ is not of Calderón type $p$ (as defined in [10] for $p>1)$.

In a forthcoming paper we will describe some other properties of the spaces $[C, \Lambda]_{\theta}$ and $[C, \Lambda]^{\theta}$.

\section{The COUPLE $(C, \Lambda)$}

Lemma 1. For each $\theta \in(0,1)$ the function $t^{\theta}$ is an element of $[C, \Lambda]^{\theta}$.

Proof. For each $t \in[0,1]$ and $z \in S$ we define the function $h(t, z)$ by setting $h(0, z)=0$ and $h(t, z)=\int_{1}^{z} t^{\zeta} d \zeta$ for $t \in(0,1]$. Thus $h(1, z)=z-1$ and $h(t, z)=$ $\frac{t^{z}-t}{\log t}$ for $t \in(0,1)$. Clearly $h$ is an analytic function of $z$ for each fixed $t \in[0,1]$ and $h(\cdot, z) \in C$ for each fixed $z \in S$ and also satisfies

$$
\|h(\cdot, z)\|_{C} \leq|z-1|
$$

(since $\left|t^{\zeta}\right| \leq 1$ for all $\zeta \in S$ and $\left.t \in(0,1]\right)$. Similarly

$$
\left\|h(\cdot, z)-h\left(\cdot, z^{\prime}\right)\right\|_{C} \leq\left|z-z^{\prime}\right|
$$

for all $z$ and $z^{\prime} \in S$. Thus we see that $h(z)=h(\cdot, z)$ is a continuous map of $S$ into $C[0,1]$ and it is also clear that it is also analytic when considered as a vector valued (i.e. $C[0,1]$ valued) function of $z$. We observe that $\frac{\partial}{\partial z} h(t, z)=t^{\theta}$ for $z=\theta$ and so also $\frac{\partial}{\partial z} H(t, \theta)=t^{\theta}$ where the function $H$ is defined by $H(t, z)=e^{(z-\theta)^{2}} h(t, z)$.

To complete the proof we must show that $H \in \overline{\mathcal{F}}(C, \Lambda)$. Let us first see that $h$ is "almost" in $\overline{\mathcal{F}}(C, \Lambda)$. For any real $\alpha$ and $\beta$ we note that

$$
\|h(\cdot, j+i \beta)-h(\cdot, j+i \alpha)\|_{C} \leq|\beta-\alpha|
$$

for $j=0,1$ (cf. (3)). Now

$$
h(t, 1+i \beta)-h(t, 1+i \alpha)=\int_{1+i \alpha}^{1+i \beta} t^{\zeta} d \zeta
$$

and

$$
\begin{aligned}
\left|\int_{1+i \alpha}^{1+i \beta} t^{\zeta}-s^{\zeta} d \zeta\right| & =\left|\int_{\alpha}^{\beta} t^{1+i \sigma}-s^{1+i \sigma} d \sigma\right|=\left|\int_{\alpha}^{\beta} \int_{s}^{t}(1+i \sigma) \tau^{i \sigma} d \tau d \sigma\right| \\
& \leq|\beta-\alpha||t-s|(1+\max (|\alpha|,|\beta|) .
\end{aligned}
$$


Consequently (using (4) also) we obtain that

$$
\|h(\cdot, 1+i \beta)-h(\cdot, 1+i \alpha)\|_{\Lambda} \leq|\beta-\alpha|(1+\max (|\alpha|,|\beta|) .
$$

In particular, for $\beta=0$, we have

$$
\|h(\cdot, 1+i \alpha)\|_{\Lambda} \leq|\alpha|(1+|\alpha|) .
$$

Now, setting $v(z)=e^{(z-\theta)^{2}}$, we see that, for $j=0,1$,

$$
|v(j+i \beta)| \leq e^{1-\beta^{2}}
$$

and, if $|\alpha-\beta| \leq 1$,

$$
|v(j+i \alpha)-v(j+i \beta)| \leq 2(2+|\beta|) e^{1-\left(\min (|\beta|-1,|\beta|)^{2}\right.}|\beta-\alpha| .
$$

Then

$$
\begin{aligned}
\|H(1+i \alpha)-H(1+i \beta)\|_{\Lambda} \leq & \|(v(1+i \alpha)-v(1+i \beta)) h(1+i \alpha)\|_{\Lambda} \\
& +\|v(1+i \beta)(h(1+i \alpha)-h(1+i \beta))\|_{\Lambda},
\end{aligned}
$$

and, by (8), (6), (7) and (5) this expression is dominated by $M|\beta-\alpha|$ for all real $\alpha$ and $\beta$ satisfying $|\beta-\alpha| \leq 1$, where $M$ is an absolute constant. But in fact this immediately implies that the same estimate

$$
\|H(1+i \alpha)-H(1+i \beta)\|_{\Lambda} \leq M|\beta-\alpha|
$$

also holds without the restriction $|\beta-\alpha| \leq 1$.

Similarly

$$
\|H(i \alpha)-H(i \beta)\|_{C} \leq\|(v(i \alpha)-v(i \beta)) h(i \alpha)\|_{C}+\|v(i \beta)(h(i \alpha)-h(i \beta))\|_{C},
$$

and, by (8), (2), (7) and (4) we obtain that

$$
\|H(i \alpha)-H(i \beta)\|_{C} \leq M^{\prime}|\beta-\alpha|
$$

for all real $\alpha$ and $\beta$ and an absolute constant $\mathrm{M}^{\prime}$. This completes the verification that $H \in \overline{\mathcal{F}}(C, \Lambda)$.

From the standard inclusions between real and complex interpolation spaces and the fact $\left([14],\left[3\right.\right.$, p. 301]) that $K(t, f ; C, \Lambda)$ is equivalent to $\min (1, t)\|f\|_{C}+\omega(t, f)$ it follows that

$$
[C, \Lambda]_{\theta} \subset[C, \Lambda]^{\theta} \subset(C, \Lambda)_{\theta, \infty}=\Lambda_{\theta} .
$$

Since $\Lambda$ is dense in $[C, \Lambda]_{\theta}$ and the closure of $\Lambda$ in $\Lambda_{\theta}$ is $\lambda_{\theta}$, we also have that $[C, \Lambda]_{\theta} \subset \lambda_{\theta}$.

Now, since $t^{\theta}$ is an element of $\Lambda_{\theta}$ whose modulus of continuity has maximal growth rate, it seems natural to conjecture, in the light of Lemma 1 , that $[C, \Lambda]^{\theta}=$ $\Lambda_{\theta}$. Obviously $[C, \Lambda]_{\theta}$ cannot coincide with $\Lambda_{\theta}$ for the simple reason that $\Lambda$ is not dense in $\Lambda_{\theta}$. However it would seem natural to conjecture that $[C, \Lambda]_{\theta}=\lambda_{\theta}$.

We shall soon see that both these conjectures are false. 
Lemma 9. For each $\theta \in(0,1)$ the Fourier coefficients $\gamma_{n}=\int_{0}^{1} e^{-2 \pi n i t} g(t) d t$ of each function $g \in[C, \Lambda]^{\theta}$ satisfy

$$
\left(\sum_{n=-\infty}^{\infty}\left|\gamma_{n}\right|^{2}|2 \pi n|^{2 \theta}\right)^{1 / 2} \leq\|g\|_{[C, \Lambda]^{\theta}} .
$$

Proof. $C[0,1]$ is continuously embedded in $L^{2}[0,1], \Lambda$ is continously embedded in the Sobolev space $W^{1,2}[0,1]$ and both embeddings have norm 1 . Thus the linear map $\mathbf{F}$ which maps each integrable function on $[0,1]$ to the sequence of its Fourier coefficients maps $C$ into $\ell^{2}$ with norm 1 and also maps $\Lambda$ into the weighted $\ell^{2}$ space $\ell^{2}(\{2 \pi|n|\})$ with norm 1 . Thus $\mathbf{F}$ must be a norm 1 map of $[C, \Lambda]^{\theta}$ into $\left[\ell^{2}, \ell^{2}(\{2 \pi|n|\})\right]^{\theta}=\left[\ell^{2}, \ell^{2}(\{2 \pi|n|\})\right]_{\theta}=\ell^{2}\left(\left\{(2 \pi|n|)^{\theta}\right\}\right)$.

Corollary 10. $[C, \Lambda]^{\theta} \neq \Lambda_{\theta}$ and $\lambda_{\theta} \neq[C, \Lambda]_{\theta}$ for each $\theta \in(0,1)$.

Proof. The function $g(x)=\sum_{n=-\infty}^{\infty} \gamma_{n} e^{2 \pi i n x}=\sum_{k=1}^{\infty} 2^{-k \theta} e^{2 \pi i 2^{k} x}$ cannot be in $[C, \Lambda]^{\theta}$ since $\sum_{n=-\infty}^{\infty}\left|\gamma_{n}\right|^{2}(2 \pi|n|)^{2 \theta}=\sum_{k=1}^{\infty}\left|2^{-k \theta}\right|^{2}\left(2 \pi\left|2^{k}\right|\right)^{2 \theta}$ is of course divergent. On the other hand it is well known and easy to check that $g \in \Lambda_{\theta}$. (To do this observe that $|g(x+h)-g(x)| \leq \sum_{k=1}^{\infty} 2^{-k \theta}\left|e^{2 \pi i 2^{k} h}-1\right| \leq \sum_{k=1}^{N} 2^{-k \theta}\left|2 \pi 2^{k} h\right|+$ $2 \sum_{k=N+1}^{\infty} 2^{-k \theta} \leq C\left(2^{N(1-\theta)}|h|+2^{-N \theta}\right)$ for some constant $C$ depending only on $\theta$. Then choose $N$ so that $2^{-N} \sim|h|$.)

Now consider the functions $g_{N}$ for $N=1,2, \ldots$ where $g_{N}$ is obtained by convolving $g$ with the $N$ th order Fejér kernel. These are clearly in $\lambda_{\theta}$ and $\left\|g_{N}\right\|_{\lambda_{\theta}} \leq\|g\|_{\Lambda_{\theta}}$ for each $N$. If $\lambda_{\theta}$ coincides with $[C, \Lambda]_{\theta}$, then $\left\{g_{N}\right\}$ must be a bounded sequence in $[C, \Lambda]^{\theta}$ and so the Fourier coefficients $\gamma_{n N}$ of $g_{N}$ must have the property that $\sum_{n=-\infty}^{\infty}\left|\gamma_{n N}\right|^{2}(2 \pi|n|)^{2 \theta}$ is uniformly bounded for all $N$. But this is clearly false and so $\lambda_{\theta} \neq[C, \Lambda]_{\theta}$.

Corollary 11. The couple $(C, \Lambda)$ is not a Calderón couple.

Proof. The function $g$ defined above satisfies $K(t, g ; C, \Lambda) \leq M K(t, f ; C, \Lambda)$ for all $t>0$, where $M$ is some constant and $f$ is the function $f(x)=x^{\theta}$. However there cannot exist a bounded linear operator $T:(C, \Lambda) \rightarrow(C, \Lambda)$ such that $T f=g$.

The COuples $\left(C, \Lambda_{\alpha}\right)$ AND $\left(\Lambda_{\alpha}, \Lambda\right)$ FOR $0<\alpha<1$

Since $(C, \Lambda)_{\theta, \infty}=\Lambda_{\theta}$ for each $\theta \in(0,1)$, it follows from [9] that the couple $\left(\Lambda_{\theta_{0}}, \Lambda_{\theta_{1}}\right)$ is a Calderón couple whenever $0<\theta_{0}<\theta_{1}<1$. As we have seen in the previous section, this is false if both $\theta_{0}=0$ and $\theta_{1}=1$. We now extend the argument given there to show that it is also false when either $0=\theta_{0}$ or $\theta_{1}=1$, i.e. for each of the couples $\left(C, \Lambda_{\alpha}\right)$ and $\left(\Lambda_{\alpha}, \Lambda\right)$ when $\alpha$ is a fixed number in $(0,1)$. We shall first consider the couple $\left(C, \Lambda_{\alpha}\right)$.

The first step is a modification of Lemma 1:

Lemma 12. For each $\theta \in(0,1)$ the function $t^{\alpha \theta}$ is an element of $\left[C, \Lambda_{\alpha}\right]^{\theta}$.

Proof. Although it is not known in general whether the upper complex method $[\cdot, \cdot]^{\theta}$ satisfies reiteration theorems as does the method $[\cdot, \cdot]_{\theta}(\mathrm{cf} .[7],[8])$ we do have inclusions in the "easy" direction: In particular, for any Banach couple $\left(A_{0}, A_{1}\right)$,

$$
\left[A_{0}, A_{1}\right]^{\alpha \theta} \subset\left[A_{0},\left[A_{0}, A_{1}\right]^{\alpha}\right]^{\theta}
$$


with continuous (norm one) inclusion. This seems rather obvious from the definitions, but there are some technicalities to be checked. To prove (13) let $a$ be an arbitrary element of $\left[A_{0}, A_{1}\right]^{\alpha \theta}$ with norm less than 1 . Then $a=h^{\prime}(\alpha \theta)$ for some element $h \in \overline{\mathcal{F}}\left(A_{0}, A_{1}\right)$ with norm less than 1 . Our goal will be to check that the function $f$ defined by $f(z)=\frac{1}{\alpha} h(\alpha z)$ is an element of $\overline{\mathcal{F}}\left(A_{0},\left[A_{0}, A_{1}\right]^{\alpha}\right)$ with norm less than 1 , and this of course will yield (13). That is, we have to verify that $f$ satisfies conditions i) - iv) of [7] Section 5, p. 115 for the couple $\left(B_{0}, B_{1}\right)=\left(A_{0},\left[A_{0}, A_{1}\right]^{\alpha}\right)$ :

Using a remark of Calderón (cf. [1] p. 776), we can assume that $h(j+i y)$ assumes values in $A_{j}$ for $j=0,1$ and all real $y$. Then we also have that $\|h(j+i y)\|_{A_{j}} \leq$ $\|h(j)\|_{A_{j}}+|y|$. It follows easily that $e^{z^{2}} h(z) \in \mathcal{F}\left(A_{0}, A_{1}\right)$. More precisely, for each $\delta>0$, the function $h_{\delta}$ defined by $h_{\delta}(z)=\frac{e^{\delta z^{2}}}{1+z} h(z)$ is in $\mathcal{F}\left(A_{0}, A_{1}\right)$ and $\left\|h_{\delta}\right\|_{\mathcal{F}\left(A_{0}, A_{1}\right)} \leq C$ for some constant $C$ independent of $\delta$, for all $\delta \in(0,1)$. Let $\left\{g_{n}\right\}$ be a sequence in the class $\mathcal{G}\left(A_{0}, A_{1}\right)$ which tends to $h_{\delta}$ in $\mathcal{F}\left(A_{0}, A_{1}\right)$ (cf. [7] Section 9.2 , p. 116). Obviously $g_{n}(\alpha z)$ tends to $h_{\delta}(\alpha z)$ in $\mathcal{F}\left(B_{0}, B_{1}\right)$ and

$$
\begin{aligned}
\left\|h_{\delta}(\alpha \cdot)\right\|_{\mathcal{F}\left(B_{0}, B_{1}\right)} & \leq \lim _{n \rightarrow \infty}\left\|g_{n}(\alpha \cdot)\right\|_{\mathcal{F}\left(B_{0}, B_{1}\right)} \\
& \leq \lim _{n \rightarrow \infty}\left\|g_{n}(\cdot)\right\|_{\mathcal{F}\left(A_{0}, A_{1}\right)}=\left\|h_{\delta}\right\|_{\mathcal{F}\left(A_{0}, A_{1}\right)} \leq C .
\end{aligned}
$$

Thus, by standard properties of the spaces $\mathcal{F}$, we have $\left\|h_{\delta}(\alpha z)\right\|_{B_{0}+B_{1}} \leq C$ for all $\delta \in(0,1)$ and all $z \in S$. From this we deduce that $\|f(z)\|_{B_{0}+B_{1}} \leq c(1+|z|)$ for all $z \in S$ and some constant $c$, i.e. condition i) of [7]. The verifications of the remaining conditions ii), iii) and iv) are more straightforward, using the fact that the functions

$$
u_{\delta}(z):=e^{\delta z^{2}-\delta} \frac{h(z+i s)-h(z+i t)}{s-t}
$$

are elements of the open unit ball of $\mathcal{F}\left(A_{0}, A_{1}\right)$ for all real $s \neq t$ and all $\delta>0$. It also follows that $\|f\|_{\overline{\mathcal{F}}\left(B_{0}, B_{1}\right)}<1$. We leave the details to the reader. (Observe that the above argument actually gives the stronger result $\left[A_{0}, A_{1}\right]^{\alpha \theta} \subset\left[A_{0},\left[A_{0}, A_{1}\right]_{\alpha}\right]^{\theta}$.)

We can now apply Lemma 1 and (13) to the particular couple $(C, \Lambda)$ to see that the function $t^{\alpha \theta}$ is an element of $[C, \Lambda]^{\alpha \theta} \subset\left[C,[C, \Lambda]^{\alpha}\right]^{\theta} \subset\left[C,(C, \Lambda)_{\alpha, \infty}\right]^{\theta}=$ $\left[C, \Lambda_{\alpha}\right]^{\theta}$, which completes the proof.

Next we obtain a variant of Lemma 9:

Lemma 14. For each $\theta \in(0,1)$ the Fourier coefficients $\gamma_{n}=\int_{0}^{1} e^{-2 \pi n i t} g(t) d t$ of each function $g \in\left[C, \Lambda_{\alpha}\right]^{\theta}$ satisfy

$$
\left(\sum_{n=-\infty}^{\infty}\left|\gamma_{n}\right|^{q}(2 \pi|n|)^{q \alpha \theta}\right)^{1 / q} \leq C\|g\|_{\left[C, \Lambda_{\alpha}\right]^{\theta}}
$$

where $1 / q=(1-\theta) / 2$ and $C$ is a constant depending only on $\alpha$ and $\theta$.

Proof. Applying real interpolation to the Fourier transform $\mathbf{F}$ we obtain that it maps $\Lambda_{\alpha}=(C, \Lambda)_{\alpha, \infty}$ boundedly into $\left(\ell^{2}, \ell^{2}(\{2 \pi|n|\})\right)_{\alpha, \infty}$ and so it must map $\left[C, \Lambda_{\alpha}\right]^{\theta}$ boundedly into $\left[\ell^{2},\left(\ell^{2}, \ell^{2}(\{2 \pi|n|\})\right)_{\alpha, \infty}\right]^{\theta}$. By the reflexivity of $\ell^{2}$ and $[7]$ Section 9.5, pp. 117-118 this space coincides with $\left[\ell^{2},\left(\ell^{2}, \ell^{2}(\{2 \pi|n|\})\right)_{\alpha, \infty}\right]_{\theta}$. By 
[11] Theorem 1.2, pp. 905-6 this space in turn coincides with $\left(\ell^{2}, \ell^{2}(\{2 \pi|n|\})\right)_{\alpha \theta, q}$ where $1 / q=(1-\theta) / 2$. Since $q>2$, we deduce that

$$
\left(\ell^{2}, \ell^{2}(\{2 \pi|n|\})\right)_{\alpha \theta, q} \subset\left(\ell^{q}, \ell^{q}(\{2 \pi|n|\})\right)_{\alpha \theta, q},
$$

and this latter space is of course the same as $\ell^{q}\left(\left\{|2 \pi n|^{\alpha \theta}\right\}\right)$ (cf. [2] Theorem 5.4.1, p. 115). This implies (15) and completes the proof.

Now replacing $\theta$ by $\alpha \theta$ in the definition of the function $g$ used above in Corollary 10, we obtain a function $g_{\alpha}$ which is in $\Lambda_{\alpha \theta}$ but clearly not in $\left[C, \Lambda_{\alpha}\right]^{\theta}$. The second part of the proof of Corollary 10 can also be immediately adapted to show that $\lambda_{\alpha \theta} \neq\left[C, \Lambda_{\alpha}\right]_{\theta}$.

Finally let us show that $\left(C, \Lambda_{\alpha}\right)$ is not a Calderón couple: Observe that $g_{\alpha}$ satisfies $K\left(t, g_{\alpha} ; C, \Lambda\right) \leq M K\left(t, f_{\alpha} ; C, \Lambda\right)$ for all $t>0$, where $M$ is some constant and $f_{\alpha}$ is the function $f(x)=x^{\alpha \theta}$. For any couple $\left(A_{0}, A_{1}\right)$ it is well known (and easy to check) that the $K$-functional for the couple $\left(A_{0},\left(A_{0}, A_{1}\right)_{\alpha, \infty}\right)$ is given to within equivalence by

$$
K\left(t, a ; A_{0},\left(A_{0}, A_{1}\right)_{\alpha, \infty}\right) \sim t \sup _{s>t^{1 / \theta}} s^{-\theta} K\left(s, a ; A_{0}, A_{1}\right) .
$$

(Cf. also Remark 2.1 of [12] p. 183 and [3].) This implies that for some constant $M^{\prime}$ we have $K\left(t, g_{\alpha} ; C, \Lambda_{\alpha}\right) \leq M^{\prime} K\left(t, f_{\alpha} ; C, \Lambda_{\alpha}\right)$ for all $t>0$. However there cannot exist a bounded linear operator $T:\left(C, \Lambda_{\alpha}\right) \rightarrow\left(C, \Lambda_{\alpha}\right)$ such that $T f_{\alpha}=g_{\alpha}$. Consequently $\left(C, \Lambda_{\alpha}\right)$ is not Calderón.

We can now deal with the second couple $\left(\Lambda_{\alpha}, \Lambda\right)$ by easy modifications of the preceding discussion. Exactly analogously to the proof of Lemma 12 we can show that $\left[A_{0}, A_{1}\right]^{\alpha(1-\theta)+\theta} \subset\left[\left[A_{0}, A_{1}\right]^{\alpha}, A_{1}\right]^{\theta}$. (This can also be deduced from (13) and the general formula $\left[X_{0}, X_{1}\right]^{\sigma}=\left[X_{1}, X_{0}\right]^{1-\sigma}$.) It is convenient to set $\beta=$ $\alpha(1-\theta)+\theta$. It follows that the function $t^{\beta}$ is an element of $\left[\Lambda_{\alpha}, \Lambda\right]^{\theta}$. Then minor modifications of the proof of Lemma 14 show that $\mathbf{F}$ maps $\left[\Lambda_{\alpha}, \Lambda\right]^{\theta}$ into $\ell^{q}\left(\left\{|2 \pi n|^{\beta}\right\}\right)$ with $q=2 / \theta$. We deduce that the function $\sum_{k=1}^{\infty} 2^{-k \beta} e^{2 \pi i 2^{k} x}$ is in $\Lambda_{\beta} \backslash\left[\Lambda_{\alpha}, \Lambda\right]^{\beta}$ and use this function and the function $t^{\beta}$ to show that $\left(\Lambda_{\alpha}, \Lambda\right)$ is not Calderón.

\section{ACKNOWLEDGEMENT}

The second author thanks the Lady Davis Foundation and the Technion for support and hospitality during a visit to Haifa when most of this research was carried out.

\section{REFERENCES}

1. J. Bergh, On the relation between the two complex methods of interpolation, Indiana Univ. Math. J. 28 (1979), 775-778. MR 80f:46062

2. J. Bergh and J. Löfström, Interpolation spaces. An introduction, Grundlehren Math. Wiss., vol. 223, Springer, Berlin, Heidelberg, and New York, 1976. MR 58:2349

3. Y. Brudnyi and N. Krugljak, Interpolation functors and interpolation spaces, North-Holland, Amsterdam, New York, Oxford, and Tokyo, 1991. MR 93b:46141

4. Y. Brudnyi and A. Shteinberg, Calderón couples of Lipschitz spaces, J. Functional Analysis 131 (1995), 459-498. CMP 95:16

5. _ Calderón constants of finite dimensional couples, Israel J. Math. (to appear).

6. T. Bychkova, Couples without C-properties, Investigations in the Theory of Functions of Several Variables, Yaroslavl, 1990, pp. 18-51. 
7. A. P. Calderón, Intermediate spaces and interpolation, the complex method, Studia Math. 24 (1964), 113-190. MR 29:5097

8. M. Cwikel, Complex interpolation, a discrete definition and reiteration, Indiana Univ. Math. J. 27 (1978), 1005-1009. MR 80h:46118

9. Monotonicity properties of interpolation spaces II, Ark. Mat. 19 (1981), 123-136. MR 83a:46082

10. $\quad K$-divisibility of the K-functional and Calderón couples, Ark. Mat. 22 (1984), 39-62. MR 85m:46074

11. M. Cwikel and Y. Sagher, Relations between real and complex interpolation spaces, Indiana Univ. Math. J. 36 (1987), 905-912. MR 88m:46083

12. T. Holmstedt, Interpolation of quasi-normed spaces, Math. Scand. 26 (1970), 177-199. MR 54:3440

13. V. I. Ovchinnikov, Interpolation properties of fractional BMO space, Abstracts of Proceedings of the 13th All-Union School in Operator Theory in Functional Spaces, Kuibyshev, 1988.

14. J. Peetre, Exact interpolation theorems for Lipschitz continuous functions, Richerche Mat. 18 (1969), 239-259. MR 42:841

Department of Mathematics, Technion Israel Institute of Technology, Haifa, 32000 ISRAEL

E-mail address: mcwikel@techunix.technion.ac.il

Faculty of Mathematics and Computer Science, A. Mickiewicz University, Matejk 48/49, 60-769 Poznań, Poland

E-mail address: mastylo@math.amu.edu.pl 